Soviet Union's "Northern Sea Route" in the account of Dr Terence Armstrong of the Scott Polar Research Institute, who has made a detailed study going back many years. This has been actively operated by the Soviet Union for many years and the route's administration had an almost autonomous existence until the 1930s purges. Some 400 ships ply in the area transporting $2 \frac{1}{2}$ million tons of freight annually. Recently a large programme of 24 specially designed medium-sized icebreakers has been laid down in Finland for the route, quite apart from the completion of the hugely powerful new atom-powered Soviet-built icebreaker
Arktika, thought to be capable of 75,000 horsepower. In recent seasons efforts have been made to extend the duration of navigation, limited for the full 'through' route of $2 \frac{1}{2}$ months. A complete convoy of 13 ships actually struggled through as late as midJanuary in 1972 but a policy speech by the Transport minister subsequently indicated that the experiment proved that the route could be kept open into December but not beyond (giving a September to Christmas season).

The various moves including the large new icebreaker-building programme suggests that the Northern sea route continues to have an important future despite the impression that it has been losing ground to the "southern" rail route out of Siberia where many extensions and new links have been built in recent years. The freight tonnage of the Northern sea route is holding up well, despite the increased bulk handled by the railway. Dr Armstrong points out that the sea route was developed originally for strategic reasons that never materialised. Nevertheless, having invested heavily in a system in advance of need, he concludes that the Soviet administration is loth to abandon it and expects the Northern Sea route to justify itself until the end of the century.

\title{
correspondence
}

\section{The reward system}

SIR,-A basic fallacy of your Editorial "The Reward System Needs Overhauling" of March 271975 is that Fellowships of the Royal Society and Nobel Prizes are not rewards but awards. They are not something a reasonable scientist can ever expect to receive as a matter of course for professional work even of the greatest merit, but a happy chance in which one can rejoice if it should happen to oneself or one's colleages.

Of course Nobel Prizes "are clearly not enough to go round". Why should they be? They are not a matter of right, a sort of career-grade for the top scientist, but an extra provided by the posthumous generosity of one man. Strictly speaking, their award is the private business of the Nobel Committee and no one else. Admittedly this Committee would be prudent to take account of public standards if confidence in the awards is to be maintained, but your criticism on this score is nullified by your own statement that Nobel Prizes "confer an unreasonable amount of credit on their recipients". Moreover it is invidious to critise the Nobel Committee for confining their awards "to a restricted number of subjects" when they have broadened their interpretation, within the terms of reference by which they are bound, to include astronomy.

It would have been best if the attack by Sir Fred Hoyle on these astronomical awards had been treated with the contempt of silence. Now that this silence has been broken, it needs to be said that whatever the occasion may have been, these Nobel Prizes are justified at least as much by decades of distinguished contribution to Radio Astronomy and Cosmology as by any single discovery. Sir Martin Ryle and Professor Hewish had created the physical facilities that made possible the original observations of pulsars, and the background of knowledge that enabled these observations to be interpreted. Mrs Burnell has a secure place in history as the Tombaugh of the discovery, and her credit has been enhanced by the calmness of the remarks she is reported to have made.

An important lesson from the whole unfortunate matter is that even very distinguished theoreticians may be profoundly ignorant of the realities of discovery in experimental science, and this calls into question the heavy reliance that has been placed on theoretical astronomers in advising SRC and other bodies on strategies for British astronomy.

It may be true that some laboratories make the "pursuit of Nobel Prizes ... the ultimate aim". If so, the more fools they; in seeking external awards they are missing the true rewards of science. One expects scientists to have something better to do than to display the envy of socialites denied admittance to an exclusive club. If we are indeed seeing "an early shot in a battle which will set scientists against each other in years to come", it will be because they have allowed themselves to be led by journalists whose stock in trade is to whip up "controversy". As one who has neglible chance of ever becoming, let alone deserving, an FRS or a Nobel Laureate, I shall continue to live without envy of those on whom this fortune falls, and I think well enough of my colleagues to believe that the majority of them will do the same.

University of Reading, $U K$

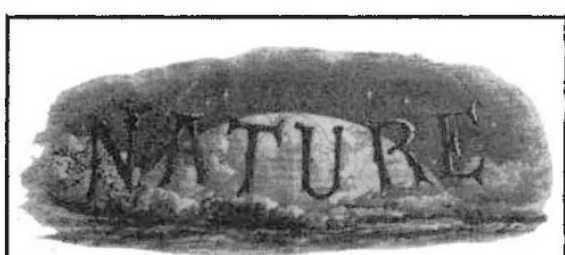

\section{A hundred years ago}

THE Rev. G. H. Hopkins gives the following method for fixing the curves which steel filings take when under the action of a bar magnet. The filings having been prepared so as to be as nearly the same size as possible, and that size very minute, are pound into a mortar, and a small quantity of finely powdered resin is added; these are stirred together until the two substances are completely mixed, and then, considerable pressure being exerted upon the pestle, they are rubbed until the resin adheres to the filings in a very fine coating. The filings can then be sprinkled as usual, and the curves formed. It is best (after the curves are formed) to heat the plane surface, glass, paper, or wood, according to convenience, over a stove or in an oven, which easily allow it to be sufficiently as well as uniformly heated. For projecting the curves on a screen, the following, we beliewe, is a very effective method. Cover the glass with thin gum-water, allow it to dry perfectly; obtain the curves on the dry gummed surface; finally, breathe on the plate: the gum is thereby softened and the curve permanently fixed. Substituting corresponding shaped pieces of paper for the magnets (a pin-hole can be used to indicate the N. pole), the curves can be covered with a second plate of glass, and thus preserved as an ordinary lantern slide

from Nature, 12, 97; 3 June, 1875 